\title{
Conformational Flexibility of the Ligand-Binding Domain Dimer in Kainate Receptor Gating and Desensitization
}

\author{
Naushaba Nayeem, ${ }^{1}$ Olga Mayans, ${ }^{2}$ and Tim Green ${ }^{1}$ \\ ${ }^{1}$ Department of Pharmacology and ${ }^{2}$ School of Biological Sciences, University of Liverpool, Liverpool L69 3BX, United Kingdom
}

\begin{abstract}
AMPA- and kainate (KA)-selective ionotropic glutamate receptors (iGluRs) respond to agonist by opening (gating), then closing (desensitizing) in quick succession. Gating has been linked to agonist-induced changes within the ligand-binding domain (LBD), and desensitization to rearrangement of a dimer formed by neighboring LBDs. To explore the role of dimer conformation in both gating and desensitization, we compared the conformational effects of two kainate receptor mutants. The first, GluK2-D776K, blocks desensitization of macroscopic current responses ("macroscopic desensitization"). The second, GluK2-M770K, accelerates macroscopic desensitization and eliminates the effects of external ions on channel kinetics. Using structures determined by x-ray crystallography, we found that in both mutants the introduced lysines act as tethered cations, displacing sodium ions from their binding sites within the dimer interface. This results in new inter-and intra-protomer contacts in D776K and M770K respectively, explaining the effects of these mutations on dimer stability and desensitization kinetics. Further, chloride binding was unaffected by the M770K mutation, even though binding of sodium ions has been proposed to promote dimer stability by stabilizing anion binding. This suggests sodium binding may affect receptor function more directly than currently supposed. Notably, we also observed a ligand-specific shift in dimer conformation when comparing LBD dimers in complex with glutamate or the partial agonist KA, revealing a previously unidentified role for dimer orientation in iGluR gating.
\end{abstract}

\section{Introduction}

AMPA and kainate (KA) ionotropic glutamate receptors (iGluRs) (non-NMDA receptors), are involved in a diverse range of physiological and pathological processes in the CNS. As potential drug targets, the conformational effects of ligand binding to these receptors have been intensively studied. Most reports have focused on the ligand-binding domain (LBD), which is formed by two noncontiguous regions ( $\mathrm{S} 1$ and S2) flanking the pore-forming domains. Crystal structures of iGluR subunit LBDs have provided insights into receptor movements during both receptor gating and desensitization. Ligands bind between the "upper" (D1) and "lower" (D2) lobes of the LBD; for some ligands movement of the lobes together ("cleft-closure") is correlated with efficacy in non-NMDA receptors (Armstrong and Gouaux, 2000; Mayer, 2006), although the basis for this is subject to debate. Isolated LBDs interact through their D1 domains to form dimers related by twofold symmetry, with the receptor tetramer assembled as an intercalating "dimer of dimers" in both AMPA (Sobolevsky et al., 2009) and kainate receptors (Das et al., 2010).

Desensitization is linked to dimer rearrangement, with the D1dimer associated with the active state, and a hinged separation of the D1-D1 interface observed in a desensitized state (Armstrong et al., 2006). Stabilization of the D1-dimer therefore attenuates desensiti-

\footnotetext{
Received Sept. 11, 2010; revised Nov. 15, 2010; accepted Dec. 22, 2010.

The rat GluK2 CDNA was a gift from Prof. Steve Heinemann (Salk Institute, La Jolla, CA). We thank Dean Madden (Dartmouth College Medical School, Hanover, NH) for helpful discussions. We are also grateful to Sonia Barbieri and Barbara Franke (School of Biological Sciences, University of Liverpool) for assistance with data collection, and to beamline staff at the DIAMOND and BESSY-II synchrotrons.

Correspondence should be addressed to Tim Green at the above address. E-mail: tpgreen@liv.ac.uk.

DOI:10.1523/JNEUROSCI.4771-10.2011

Copyright $\odot 2011$ the authors $\quad 0270-6474 / 11 / 312916-09 \$ 15.00 / 0$
}

zation (Sun et al., 2002; Chaudhry et al., 2009b). Macroscopic desensitization can be blocked by covalent cross-links (Priel et al., 2006; Weston et al., 2006) and single point-mutations (Stern-Bach et al., 1998; Nayeem et al., 2009) in both AMPA and kainate receptors. In AMPA subunits a leucine-to-tyrosine mutation increases dimer affinity by $>10^{5}$-fold through increased contacts between D1 domains (Sun et al., 2002). Macroscopic desensitization is only slowed when these interactions are recreated in kainate subunits (Weston et al., 2006; Zhang et al., 2006), but it is blocked by the charge-reversal mutation GluK2-D776K (Nayeem et al., 2009). This residue is one of a cluster of four charged-residues involved in inter-dimer contacts at the LBD apex (Fig. 1); mutation of Asp776 to lysine would be expected to break these contacts, destabilize the dimer and therefore promote, rather than block, desensitization. Further, chloride and sodium ions bind within this part of the dimer interface (Fig. 2A), and even conservative mutations in this region generally accelerate desensitization (Plested and Mayer, 2007; Wong et al., 2007; Plested et al., 2008).

We hypothesized that the GluK2-D776K phenotype results from sodium-ion displacement by the introduced lysine, because unlike wild-type (WT), GluK2-D776K is unaffected by replacement of extracellular sodium by other cations (Nayeem et al., 2009). Here we have used x-ray crystallography to determine structural models for GluK2-D776K in complex with glutamate (Glu) and KA, along with GluK2-WT and a second lysine mutant, GluK2-M770K. The latter has a contrasting phenotype of rapid desensitization coupled with ion insensitivity (Paternain et al., 2003; Wong et al., 2007). As well as revealing localized changes in side-chain conformation within the dimer interface that serve to explain the mutant phenotypes, these structures reveal unexpected mutation- and ligand-specific changes in dimer orienta- 
S1

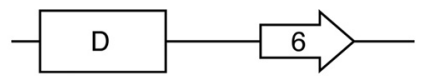

GluK2 520 T Y V R E K V I D F S K P

GluK1 535 T $Y$ V V R E GluK5 503 T A E R E K V I D F S K P GluA1 496 T L V R E E V I D F S K P

\section{GluA2 ${ }^{482} T$ L V R E E V I D F S K P}

S2

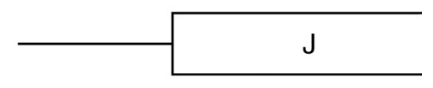

Figure 1. Alignment of apical regions of selected kainate- and AMPA-selective receptor subunits. Alignment of S1 and S2 segments around the apical cluster in the GluK2 LBD with equivalent regions in other kainate (GluK1 and GluK5) and AMPA (GluA1 and GluA2) receptor subunits. Residue numbering is for the full-length receptor, except GluA2 where it is for the mature polypeptide. $\alpha$-Helices (boxes) and a $\beta$-strand (arrow) are indicated above the sequence. In $\$ 2$, sequences of GluA 1 and GluA2 are shown for both flop (0) and flip (i) splice variants, as these differ at the sites homologous to Arg775 and Asp776. GluA2 residue 743 is changed by RNA editing from Arg to Gly (the "R/G site"). Key residues mentioned in the text are highlighted for GluK2.

A
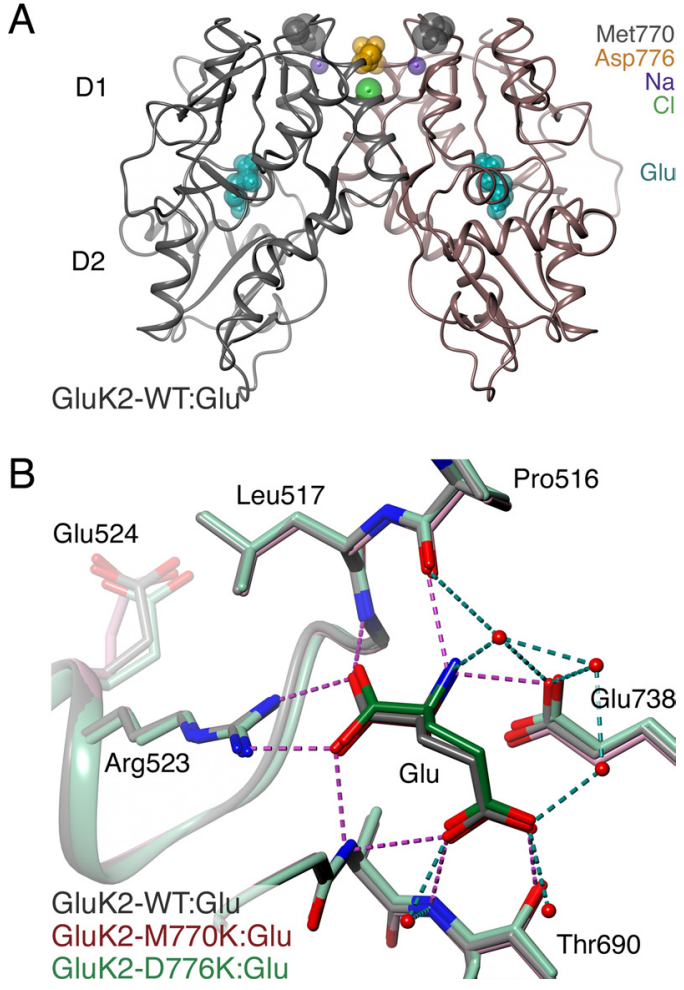

Figure 2. LBD dimer overview and ligand-binding site in GluK2-WT:Glu, GluK2-M770K:Glu, and GluK2-D776K:Glu. A, Diagram of GluK2-WT:Glu dimer, showing ligand (cyan), the residues mutated in this study (Met770, gray; Asp776, orange) and ions (sodium, purple; chloride, green) as spheres. The D1 and D2 domains are indicated. $\boldsymbol{B}$, Ligand interactions at the binding sites of GluK2-WT:Glu (gray), GluK2-M770K:Glu (pink), and GluK2-D776K:Glu (Po form; green) (all protomer B). The structures of GluK2-WT and GluK2-M770K superimpose almost exactly. Side-chains and main-chain sections that interact with the ligand Glu are displayed as sticks. Glu524, which forms part of the cation-binding pocket, is also shown. Interacting waters (from WT only) are shown as nonbonded spheres. Dashed lines represent interactions in GluK2-WT between ligand and either protein (magenta) or waters (blue-green).

tion that shed new light on gating and the actions of ions at kainate receptors.

\section{Materials and Methods}

Protein purification and crystallization. Construction, mutagenesis and expression of the GluK2 pET21a plasmid was as described previously
(Nayeem et al., 2009), with two changes: Expression was auto-induced (in Miller's LB plus $0.5 \%$ glycerol, $0.05 \%$ D-glucose, $0.2 \% \alpha$-lactose, $25 \mathrm{~mm}$ $\mathrm{Na}_{2} \mathrm{HPO}_{4}, 25 \mathrm{~mm} \mathrm{KH}_{2} \mathrm{PO}_{4}, 25 \mathrm{~mm} \mathrm{NH}_{4} \mathrm{SO}_{4}, 0.2$ $\mathrm{mM} \mathrm{MgSO}_{4}, 0.1 \mathrm{~mm} \mathrm{FeCl}_{3}$ ) (adapted from Studier, 2005) for $20 \mathrm{~h}$ at $27^{\circ} \mathrm{C}$, and terminal tags were removed before gel filtration by thrombin digestion. Constructs therefore comprised Ser429-Lys544 (the S1 domain), a GlyThr linker and Pro667-Glu806 (the S2 domain), plus an $\mathrm{N}$-terminal Gly and C-terminal ProArg from the thrombin sites. Protein was stored at $4^{\circ} \mathrm{C}$ in 25 mM HEPES, pH 7.5, 5\% glycerol, $150 \mathrm{~mm} \mathrm{NaCl}$, with $5 \mathrm{~mm}$ glutamate (crystallization buffer). GluK2-D776K oligomerization state was determined for samples dialyzed into crystallization buffer containing either $\mathrm{NaCl}, \mathrm{NaF}$, or $\mathrm{NaNO}_{3}$ by gel filtration on a Superdex 200 10/300 column. Crystals were grown in hanging-drops from GluK2 LBD constructs $(6-12 \mathrm{mg} / \mathrm{ml})$, mixed 1:1 with reservoir solution containing PEG 4000 (polyethylene glycol 4000 ), $0-9 \%$ propan-2-ol (IPA), and $80 \mathrm{~mm}$ sodium acetate. Thin plates grew in $1-3$ weeks at $22^{\circ} \mathrm{C}(27 \%$ PEG for WT and M770K; 21\% PEG for D776K). Kainate complexes were obtained by either cocrystallization with $1 \mathrm{~mm}$ KA (GluK2-D776K) or soaking Glu-complexed crystals (in 2.5-5 mm KA) for 1-2 weeks in sitting drops (GluK2-WT and GluK2$\mathrm{M} 770 \mathrm{~K})$. All crystals were cryoprotected by brief immersion in reservoir solution containing 17.5-27.5\% PEG 400 and flash frozen in liquid $\mathrm{N}_{2}$.

$X$-ray diffraction and data processing. Diffraction data were collected at $100 \mathrm{~K}$ at energies between 12.0 and $13.5 \mathrm{keV}$ ( $8 \mathrm{keV}$ for anomalous data) at beamlines I02 (M770K-Glu, M770K-KA, D776K-KA) and I03 (WTGlu, WT-KA, D776K-Glu-Po) at Diamond and beamline MX 14-2 (D776K-Glu-Io) at BESSY-II synchrotrons. Images were processed using XDS and XSCALE (Kabsch, 1993). Subsequent data manipulations used programs from the CCP4 suite (CCP4, 1994). Constructs crystallized in one of two unrelated crystal forms (Io and Po; Table 1). Initial phases were determined by molecular replacement with phaser (McCoy et al., 2007), using protomer A from GluK2 WT-Glu LBD [Protein Data Bank (PDB) accession 1S50) (Mayer, 2005) as a search model. For GluK2D776K:KA only, the GluK2-D776K:Glu Po tetramer (minus ligand and solvent, and with residue 776 set to alanine) was used directly for refinement. Models were rebuilt and refined in a number of iterations using coot (Emsley et al., 2010) and phenix.refine (Adams et al., 2002). Waters were added using phenix.refine and validated visually in coot. Omitmaps were calculated as averaged kick-maps (Pražnikar et al., 2009) in phenix.refine. Residues preceding Asn430 and following Arg800 were disordered in all protomers. The traced chain started at residue Asn430 in D776K:Glu(Io, B), residue Arg431 in M770K:Glu(B), M770K:KA(B), D776K:Glu(Po, D), D776K:Glu(Io, A) and D776K:KA(D), and residue Ser432 in all others. It ended at Trp799 except for WT:Glu(B), M770K: Glu(B) and D776K:Glu(Io, A and B), where it ended at Arg800. Residues from Asp493 to Asn495 were disordered in D776K:Glu(Po, D) and D776K:KA(D) only. There were no Ramachandran outliers. Model coordinates and diffraction data for the GluK2 WT:Glu, WT:KA, M770K: Glu, M770K:KA, D776K:Glu (Io), D776K:Glu (Po), and D776K:KA structures are deposited in the Protein Data Bank. The PDB codes are 2XXR, 2XXT, 2XXU, 2XXV, 2XXW, 2XXX and 2XXY, respectively. Structures were visualized and figures produced using CCP4MG with the exception of electrostatic surface potentials. Electrostatic calculations were performed for protein alone in the absence of bound ions using the MacPyMol (www.sourceforge.net) plug-in to the APBS program (Baker et al., 2001). The default dielectric values for protein (80) and solvent (2) were used, with $150 \mathrm{~mm}$ monovalent ion concentration. PISA (Krissinel and Henrick, 2007) was used to characterize inter-protomer interfaces. Domain movements were analyzed using dyndom (Hayward and Berendsen, 1998) from the CCP4 package. Ligand-induced cleft-closure was analyzed by pairwise comparison of single protomers (excluding flexible loops between 445 and 461 and 486 and 499). To detect domain movements at the dimer interface, coordinate files were generated con- 
Table 1. Data collection and refinement statistics

\begin{tabular}{|c|c|c|c|c|c|c|c|}
\hline \multirow{2}{*}{$\begin{array}{l}\text { Dataset } \\
\text { Ligand } \\
\text { (space group) }\end{array}$} & \multicolumn{2}{|l|}{ WT } & \multicolumn{2}{|l|}{ M770K } & \multicolumn{3}{|l|}{ D776K } \\
\hline & $\begin{array}{l}\text { :Glu } \\
(I 222)\end{array}$ & $\begin{array}{l}: K A \\
(1222)\end{array}$ & $\begin{array}{l}\text { :Glu 2) } \\
(\text { (I222) }\end{array}$ & $\begin{array}{l}: K A \\
(1222)\end{array}$ & $\begin{array}{l}\text { :Glu }\left[\mathrm{P}_{0}\right] \\
\left(\mathrm{P} 2_{1} \mathrm{Z}_{1} \mathrm{Z}_{1}\right)\end{array}$ & $\begin{array}{l}\text { :Glu }[\mathrm{lo}] \\
(\mathrm{I} 222)\end{array}$ & $\begin{array}{l}: K A \\
(P 2,2,2,)\end{array}$ \\
\hline Unit cell $(a, b, c)$ & $95.3,105.5$ & $97.3,105.9$ & $95.3,104.8$ & $96.3,104.9$ & $85.6,101.3$ & $93.7,106.8$ & $85.7,99.4$ \\
\hline$(\AA)$ & 114.3 & 113.5 & 114.2 & 113.6 & 125.7 & 112.9 & 124.6 \\
\hline Resolution $(\AA)^{*}$ & $\begin{array}{c}19.4-1.60 \\
(1.64-1.60)\end{array}$ & $\begin{array}{c}19.7-1.90 \\
(1.95-1.90)\end{array}$ & $\begin{array}{c}19.9-1.50 \\
(1.54-1.50)\end{array}$ & $\begin{array}{c}19.9-1.70 \\
(1.74-1.70)\end{array}$ & $\begin{array}{r}19.9-2.10 \\
(2.15-2.10)\end{array}$ & $\begin{array}{c}30.0-2.30 \\
(2.36-2.30)\end{array}$ & $\begin{array}{c}19.8-3.00 \\
(3.08-3.00)\end{array}$ \\
\hline Unique reflections* & $\begin{array}{l}75,578 \\
(5509)\end{array}$ & $\begin{array}{l}44,830 \\
(3371)\end{array}$ & $\begin{array}{l}87,370 \\
(6499)\end{array}$ & $\begin{array}{l}55,807 \\
(4257)\end{array}$ & $\begin{array}{l}63,552 \\
(4671)\end{array}$ & $\begin{array}{l}25,332 \\
(1849)\end{array}$ & $\begin{array}{l}21,368 \\
(1558)\end{array}$ \\
\hline Mean redundancy* & $5.5(4.1)$ & $5.9(5.5)$ & $5.5(3.7)$ & $7.4(4.4)$ & $4.1(4.1)$ & $3.6(3.4)$ & $7.6(8.2)$ \\
\hline Completeness (\%)* & $99.5(99.7)$ & $96.5(98.7)$ & $95.7(96.6)$ & $88.1(91.5)$ & $98.5(99.7)$ & $99.2(98.3)$ & $97.5(98.7)$ \\
\hline$I / \sigma(I)^{*}$ & $18.9(2.3)$ & $12.7(2.8)$ & $16.9(2.2)$ & $15.6(2.1)$ & $15.6(2.2)$ & $13.2(2.0)$ & $14.2(3.5)$ \\
\hline$R_{\text {symm }}{ }^{*}$ & $4.9(66.5)$ & $8.7(72.7)$ & $4.8(54.5)$ & $9.1(81.3)$ & $6.2(65.0)$ & $6.5(58.2)$ & $17.7(66.5)$ \\
\hline$R_{\text {work }} / R_{\text {free }}(\%)^{\dagger}$ & $16.9 / 19.5$ & $17.2 / 21.0$ & $16.9 / 18.9$ & $16.5 / 20.4$ & $17.9 / 23.1$ & $19.1 / 24.4$ & $22.2 / 26.4$ \\
\hline Protomers (NCS dimers) & $2(1)$ & $2(1)$ & $2(1)$ & $2(1)$ & $4(2)$ & $2(1)$ & $4(2)$ \\
\hline No. of protein atoms & 4000 & 3969 & 3998 & 3977 & 7920 & 4018 & 7920 \\
\hline No. of ligand atoms & 20 & 30 & 20 & 30 & 40 & 20 & 60 \\
\hline No. of $\mathrm{Na}^{+}$ions & 2 & 2 & 2 (partial) & None & None & None & ND \\
\hline No. of $\mathrm{Cl}^{-}$ions & 1 & 1 & 1 & 1 & ND & 1 & ND \\
\hline No. of waters & 477 & 417 & 492 & 501 & 394 & 170 & none \\
\hline $\begin{array}{c}\text { Bond/angle RMSDs } \\
(\AA / \text { degree })\end{array}$ & $0.006 / 1.02$ & $0.007 / 1.06$ & $0.006 / 1.04$ & $0.007 / 1.09$ & $0.005 / 0.82$ & $0.004 / 0.76$ & $0.008 / 1.15$ \\
\hline
\end{tabular}

ND, Not determined.

*Values in parentheses represent the highest resolution shell.

${ }^{\dagger} 5.0 \%$ of reflections were excluded for calculation of $R_{\text {free. }}$.

taining the D1 domains from both protomers in a dimer (434-535 and $764-788$, minus loops as above), concatenated into a single chain.

\section{Results}

\section{The overall LBD fold is unaffected by the M770K and D776K mutations}

Structures of the GluK2-LBD were elucidated by x-ray crystallography for the M770K and D776K mutants in complex with the full-agonist glutamate. To enable direct conformational comparison, the GluK2-WT LBD structure was also determined in the same crystallographic lattice. Crystal symmetry was either I222 (Io form) or $\mathrm{P}_{1} 2_{1} 2_{1}$ (Po form), containing one or two biological dimers respectively in the asymmetric unit (Table 1). These dimers were formed through D1 domain contacts (Fig. 2A), with a similar arrangement to that observed previously for GluK2-WT [comparing our WT dimer with 3G3F, root mean square deviation $($ RMSD $)=0.39 \AA$ )] (Chaudhry et al., 2009b). Individual protomer structures in GluK2-WT:Glu, GluK2-M770K:Glu, and GluK2-D776K:Glu were virtually identical (RMSD $=0.09-0.36$ $\AA)$, with only localized changes around the mutations. In all structures glutamate was bound in the cleft between the D1 and D2 domains (Fig. 2B). The ligand conformation was essentially as described by Mayer for GluK2-WT (2005), confirming that the agonist interacted normally with the two mutants.

\section{Displacement of sodium ions by lysine in both GluK2-M770K and GluK2-D776K}

Two sodium ions and a chloride ion bind to the GluK2 LBD dimer (Fig. 2A) (Plested et al., 2008). In GluK2-WT, sodium is coordinated by the side chain carboxyl groups of Glu524 and Asp528, the main chain carbonyl groups of Glu524 and Ile527, and a solvent molecule (Fig. $3 A$ ). The two residues mutated in this study are both located adjacent to the cation-binding site; Met770 in the same protomer and Asp776 on the opposing protomer. Neither residue makes significant contacts with the ion. Met770 caps the ion-binding pocket and Asp776 forms an inter-protomer contact with Arg775 (Fig. 3B). When Met770 is replaced with a lysine in GluK2-M770K:Glu, the side-chain amine reaches toward the sodium-binding pocket (Fig. $3 A-C$ ), displacing the sodium ion and effectively acting as a surrogate cation. However, although the amine group is within $\sim 1.3 \AA$ of the sodium ion-binding site, positive difference peaks (10.2 and $5.8 \sigma$ in protomers $\mathrm{A}$ and $\mathrm{B}$ ), centered on the sodium-binding site, remained in $\left|F_{\text {obs }}-F_{\text {calc }}\right| \alpha_{\text {calc }}$ electron density maps (Fig. $3 A$ ). From the position and coordination geometry we interpreted this as partial occupancy by sodium (estimated at between 0.25 and 0.4 ). We envisage that when sodium is bound the apical end of the Lys770 side-chain becomes disordered, possibly reaching out into the solvent. In AMPA subunits the homologous residue is a lysine, and in GluA2 has been observed in a strikingly similar conformation (supplemental Fig. $1 \mathrm{~A}$, available at www.jneurosci.org as supplemental material) (Armstrong and Gouaux, 2000). There have been no reports of partial occupancy of this site by cations in AMPA subunits, but the structural similarities make it an interesting possibility.

An equivalent displacement of sodium by lysine was also apparent in both the Po and Io crystal forms of GluK2-D776K:Glu (Fig. $3 D, E)$. The introduced lysines reach across the dimer interface, interacting with the carboxyl and carbonyl groups that form the cation-binding pocket in the opposing protomer. The lysine amine group in GluK2-D776K therefore also acts as a surrogate cation. Residual electron density attributable to binding of sodium ions was not detected in either crystal form of this mutant. In contrast to GluK2-M770K, where new contacts are formed entirely within the protomer, in GluK2-D776K the new interactions are formed between protomers. This helps explain why, instead of destabilizing the dimer as might be expected, the D776K charge-exchange mutation actually increases dimer stability. This in turn underlies the observed macroscopic desensitization block.

\section{Effects of GluK2 mutants on anion binding and inter-protomer salt-bridges}

The M770K and D776K mutations differ in their effects on receptor sensitivity to external anions, with responses unaffected by 

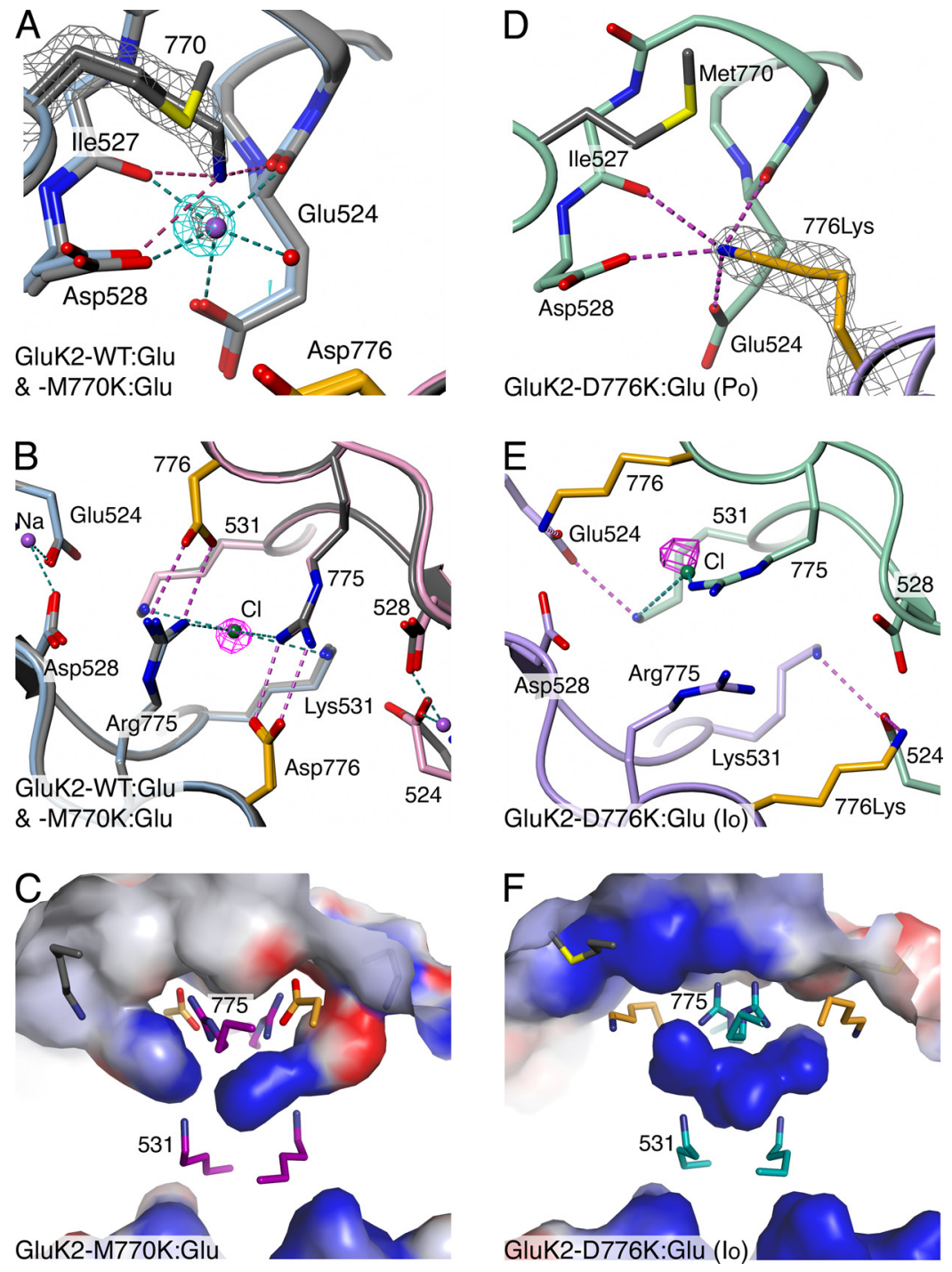

Figure 3. Comparison of apical interactions in GluK2-WT:Glu, GluK2-M770K:Glu, and GluK2-D776K:Glu. A, Superimposed view of cation-binding site in protomers A of GluK2-WT:Glu (gray) and GluK2-M770K:Glu (blue). Sodium (purple) and an interacting solvent molecule are shown for GluK2-WT only (blue-green dashes show contacts). Only the main conformer of residue 770 is shown for GluK2-M770K (magenta dashes show hydrogen-bonds). 0 mit-map (gray mesh, $\left|2 F_{\text {obs }}-F_{\text {calc }}\right| \alpha_{\text {calk }}$ contoured at $\left.1.3 \sigma\right)$ and difference-Fourier (cyan mesh, contoured at $5 \sigma$ ) electron density is shown for GluK2-M770K around lysine 770 and the sodium only. The difference-map was calculated for a model lacking sodium and with full occupancy of the main lysine 770 conformer. B, Overlay of GluK2-WT:Glu and GluK2-M770K:Glu viewed from along the dimer twofold. Sodium and chloride (green) ions and atom contacts (magenta and blue-green dashes) are displayed for GluK2-WT only. Anomalous difference-Fourier electron density (magenta mesh, contoured at $4 \sigma$ ) is displayed for GluK2-M770K. Displacement of the peak relative to the ion position results from slight non-isomorphism between datasets. $C$, Electrostatic surface potential (red, $-15 k_{b} T / e_{c}$; blue, $\left.+15 k_{b} T / e_{c}\right)$ for GluK2-M770K:Glu, calculated for protein in the absence of bound ions. The dimer interface is viewed from within protomer $B$. lon-binding site residues as shown as sticks (residues 770 and 776 colored gray and orange respectively). D, GluK2-D776K:Glu (Po crystal form) displayed as in $A$. Protomers A (purple) and B (green) are shown, with omit-map electron density for the lysine 776 only (contoured at $1.5 \sigma$ ). $\boldsymbol{E}$, $\boldsymbol{F}$, Views of GluK2-D776K:Glu (lo form), displayed as in $\boldsymbol{B}$ and $\boldsymbol{C}$, respectively.

exchange of anions in GluK2-M770K, but attenuated in GluK2D776K (Wong et al., 2007; Nayeem et al., 2009). The anionbinding site in kainate subunits is located within the dimer interface, lying between two pairs of salt bridges formed between Arg775-Asp776 and Glu524-Lys531 (Plested and Mayer, 2007). As expected, we observed a chloride ion at this position in GluK2WT:Glu (Figs. 2A,3B). Perhaps surprisingly, given its anioninsensitivity, electron density consistent with a chloride ion was also observed in GluK2-M770K:Glu. This interpretation was supported by an anomalous difference-Fourier map, which gave a clear positive peak $(6.2 \sigma)$ at this position (Fig. $3 B)$. The occu- pancies of the chloride sites in GluK2-WT and GluK2-M770K were similar (Table 1 ), indicating that chloride binding is not significantly affected by this mutation. The surface electrostatics of the ionbinding pockets were also consistent with this. In GluK2-WT the sodium and chloride-binding pockets are visible as strongly electronegative and electropositive regions respectively (supplemental Fig. $1 B$, available at www.jneurosci.org as supplemental material) (Plested et al., 2008). In GluK2-M770K the cationbinding pockets are much less electronegative, but the anion-binding pocket remains strongly electropositive (Fig. 3C).

Surprisingly, a chloride ion was not present at the equivalent site in either GluK2-D776K crystal form, but instead appears to occupy a single off-center binding site (Fig. 3E). In the Io crystal form a density-peak consistent with chloride was present in an anomalous difference map (4.3 $\sigma$; Fig. $3 E$ ). The central cavity where anions bind also retains the strong electropositive potential seen in GluK2-WT and GluK2-M770K (Fig. 3F). The pattern of difference-map density and electrostatic potential were similar within the two GluK2-D776K dimer interfaces in the Po crystal form (supplemental Fig. $1 C-G$, available at www. jneurosci.org as supplemental material). However, in this crystal form anomalous difference data could not unambiguously resolve the presence of chloride at these sites. Therefore, while it is probable that anions are also bound at this site in the Po crystal form, in the absence of a positive identification they have been modeled as waters.

The D776K mutation stabilizes the LBD dimer, increasing the $K_{\mathrm{d}}$ by over three orders of magnitude compared with GluK2-WT (Nayeem et al., 2009). To investigate whether chloride binding is required to maintain the GluK2-D776K LBD as a dimer, we performed a series of gel filtration experiments. Mobility of the GluK2-D776K LBD was unchanged in buffer containing either NaF (mean exclusion volume $\pm \mathrm{SD}$ over 3 runs $=$ $15.23 \pm 0.09 \mathrm{ml})$ or $\mathrm{NaNO}_{3}(15.13 \pm 0.13$ $\mathrm{ml})$ compared with $\mathrm{NaCl}(15.26 \pm 0.06 \mathrm{ml})$, but significantly faster than for monomeric GluK2 LBD $(16.40 \pm 0.04 \mathrm{ml} ; p<$ 0.001; one-way ANOVA, Tukey's multiple-comparisons test). Stability of the GluK2-D776K LBD dimer is therefore not dependent on the nature of the external anion, consistent with the poorly resolved chloride binding. One further change was evident in the GluK2-D776K:Glu dimers compared with GluK2-WT. As a direct consequence of the mutation, the inter-subunit saltbridge between Arg775 and Asp776 is lost. The Arg775 guanadinium groups instead adopt a stacked, anti-parallel conformation (Fig. 3E). This is similar to contacts at the homol- 
ogous site in both GluD2 (Naur et al., 2007) and GluA2 (when unedited at the R/G site) (Greger et al., 2006).

\section{Effect of kainate binding on the GluK2 LBD conformation}

The D776K mutation blocks macroscopic desensitization in responses to both full (Glu) and partial (KA and domoate) agonists (Nayeem et al., 2009). Interestingly, while the deactivation rate from peak responses to Glu was unchanged in GluK2D776K compared with GluK2-WT, the deactivation rate from peak KA responses was slowed over tenfold (Nayeem et al., 2009). This difference could result either from an indirect effect on ligand binding, or a direct effect on the dimer interface conformation. To investigate these possibilities we determined structures for GluK2-WT, GluK2-M770K, and GluK2D776K LBDs in complex with KA (Table 1). All three formed dimers, which have not been observed previously for GluK2 KA complexes. The overall fold of the protomers closely matched that of the glutamate complexes. KA bound in an analogous conformation to glutamate, with the methylethenyl group projecting out of the cleft (Fig. 4) as described previously (Mayer, 2005). Where KA had been introduced using crystal soaks (GluK2-WT:KA and GluK2-M770K:KA) effective displacement of Glu was evident in electron density maps calculated before modeling of the ligand (Fig. 4A,B). In both GluK2-M770K:KA and GluK2D776K:KA, the introduced lysines adopted the same conformations observed in the respective Glu complexes. The one difference was that in GluK2-M770K:KA there was no evidence for partial occupancy by sodium. A single chloride ion was observed in both GluK2-WT:KA and GluK2-M770K:KA (Table 1). For GluK2-D776K the resolution of the KA-complex data was insufficient to assess where or whether chloride binds.

To explore ligand-specific mutational effects we first assessed ligand-induced cleft closure. It has been shown that Glu induces a greater degree of cleft closure in GluK2 than does KA (Mayer, 2005), consistent with the action of KA as a partial agonist. We compared the extent of cleft closure in the Glu and KA complexes of GluK2-WT, GluK2-M770K, and GluK2-D776K. In each case the cleft was $\sim 4^{\circ}$ more open with KA bound (Table 2), in agreement with Mayer. Comparison of the movement of D2 centers of mass relative to D1 in protomers aligned along their principal axes (Birdsey-Benson et al., 2010), confirmed that the direction and magnitude of the ligand-induced cleft-closure movements was similar in GluK2-WT and the two mutants. In all cases the motion largely comprised movement of D2 toward D1, with only a small component of twist (supplemental Fig. 2, available at www.jneurosci. org as supplemental material).

\section{Ligand- and mutation-specific changes to the LBD} dimer conformation

We next looked for changes in the dimer interface with KA bound. The area of the interface was similar in GluK2-WT (Glu, $975 \AA^{2}$; KA, $910 \AA^{2}$ ) and GluK2-M770K (Glu, $970 \AA^{2}$; KA, 990

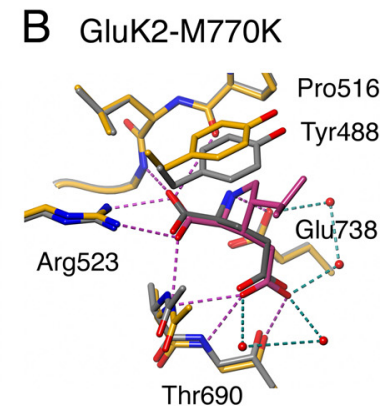

C GluK2-D776K
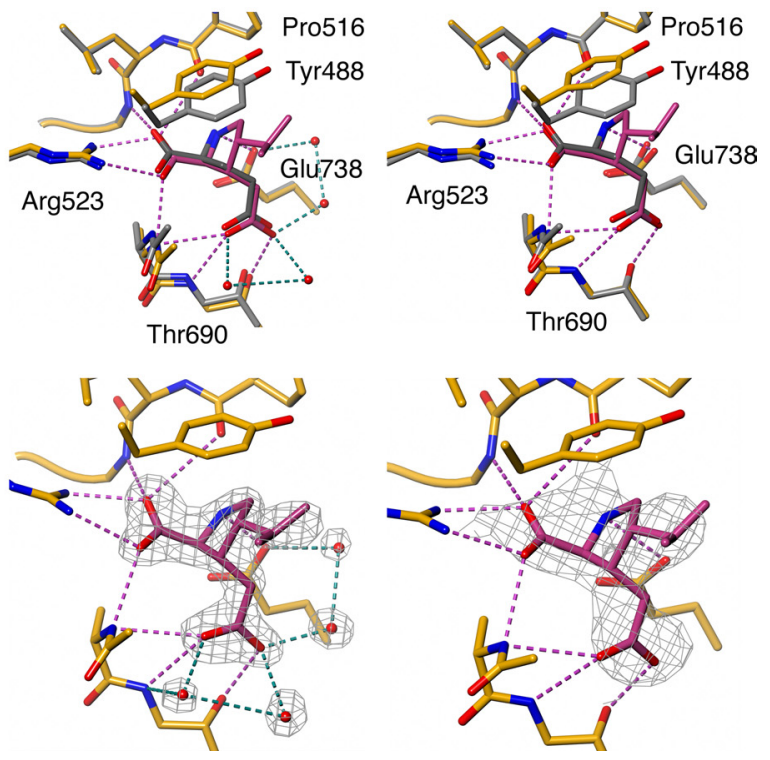

Figure 4. Kainate binding to GluK2-WT, GluK2-M770K and GluK2-D776K. A, Comparison of ligand-binding sites in Glu and KA 列 (ligand in dark gray and purple respectively). Interactions between kainate 27.5. Electron density is shown around the ligand and waters only, and contoured at $1.5 \sigma$. $\boldsymbol{B}, \boldsymbol{C}$, Equivalent views for shown. The $R$ contoured at either $1.5 \sigma(\boldsymbol{B})$ or $1.3 \sigma(\boldsymbol{C})$.

$\AA^{2}$ ). It was slightly larger in GluK2-D776K (Glu, $1050-1100 \AA^{2}$; KA, $1110-1185 \AA^{2}$ ), but this was not accompanied by changes to polar contacts beyond those described above. There were measurable changes to the overall dimer conformation, however. The distances between "linker" residues leading to and from the channel domain (i.e., Lys544 leading to M1 and Pro667 leading from M3), have been seen as a proxy for force transduction to the pore. Comparing GluK2-WT and GluK2-D776K, these linker residues were further apart in the latter structure, despite the similar degree of cleft-closure (Table 2). This resulted from movement at the dimer apex, with the side-chain rearrangements caused by the D776K mutation bringing both the $\mathrm{C} \alpha$ and $\mathrm{C} \zeta$ carbons of Arg775 closer together in GluK2-D776K (Fig. 3E; Table 2).

To quantify these dimer interface movements, we measured rigid-body shifts between the two D1 domains. Comparing the three Glu complexes (Io crystal forms), the dimer conformations in GluK2-WT and GluK2-M770K were essentially the same (Table 2). In GluK2-D776K, however, an $\sim 4^{\circ}$ shift in the dimer interface was required to superimpose the $\mathrm{D} 1$ domains with those of GluK2-WT (Fig. 5A; Table 2). The axis for this movement lay approximately along the plane of the dimer interface, resulting in a hinged motion that leads to the observed increase in linker separation and closer apical contacts (Table 2).

We performed a similar analysis to compare the dimer conformation in Glu and KA complexes. Unexpectedly, we identified ligand-specific differences in GluK2-WT and both mutants (Fig. $5 B$; Table 2). The dimer interfaces in GluK2-WT:KA and GluK2M770K:KA are rotated $\sim 2.5^{\circ}$ relative to the interface in the respective Glu complex. This movement, along axes orthogonal to the dimer twofold, acts to increase linker separation in the KA complexes (Fig. 5B; Table 2). This partly counteracts the effect of 
Table 2. Quantification of intra- and inter-protomer movements

\begin{tabular}{|c|c|c|c|c|c|c|c|}
\hline \multirow[b]{2}{*}{ GluK2 } & \multicolumn{2}{|c|}{$\begin{array}{l}\text { Cleft-closure } \\
\text { (Glu vs KA) }\end{array}$} & \multicolumn{2}{|c|}{$\begin{array}{l}\text { D1-D1 shift* } \\
\text { (1. Glu vs KA; 2. WT Glu vs mutant Glu) }\end{array}$} & \multicolumn{3}{|l|}{ Distance $(\AA)$} \\
\hline & Rotation & Translation & Rotation & Translation & Arg775 & Lys $544^{\dagger}$ & Pro66 $67^{\dagger}$ \\
\hline Mutant & $\left({ }^{\circ}\right)$ & $(\AA)$ & $\left({ }^{\circ}\right)$ & $(\AA)$ & $C \alpha-C \alpha(C \zeta-C \zeta)$ & $C \alpha-C \alpha$ & $C \alpha-C \alpha$ \\
\hline WT:Glu & $3.8 \pm 0.6$ & $0.25 \pm 0.16$ & $\begin{array}{l}\text { 1. } 2.5 \\
\text { 2. NA }\end{array}$ & $\begin{array}{l}\text { 1. } 0.09 \\
\text { 2. NA }\end{array}$ & $13.5(4.8)$ & 31.9 & 36.7 \\
\hline WT:KA & NA & NA & NA & NA & $13.2(3.8)$ & 31.4 & 35.5 \\
\hline M770K:Glu & $4.0 \pm 0.8$ & $0.19 \pm 0.16$ & $\begin{array}{l}1.2 .5 \\
2.0 .9\end{array}$ & $\begin{array}{l}\text { 1. } 0.01 \\
\text { 2. none }\end{array}$ & $13.5(4.7)$ & 31.9 & 37.0 \\
\hline M770K:KA & NA & NA & NA & NA & $13.4(4.4)$ & 31.0 & 35.3 \\
\hline D776K:Glu $[\mathrm{Io}]^{\ddagger}$ & $\begin{array}{l}A: 4.0 \\
B: 4.1\end{array}$ & $\begin{array}{l}A: 0.19 \\
B: 0.02\end{array}$ & $\begin{array}{l}\text { 1. NA } \\
\text { 2. } 3.6\end{array}$ & $\begin{array}{l}\text { 1. NA } \\
\text { 2. }-0.72\end{array}$ & $12.7(3.4)$ & 33.8 & 38.2 \\
\hline D776K:Glu $[\mathrm{Po}]^{\ddagger}$ & $\begin{array}{l}\text { A: ND } \\
B: 4.6\end{array}$ & $\begin{array}{l}\text { A: ND } \\
B:-0.18\end{array}$ & $1.2 .9 \pm 0.2$ & 1. $-0.4 \pm 0.01$ & AB: $13.1(4.1)$ & $A B: 36.6$ & $A B: 40.4$ \\
\hline & $\begin{array}{l}C: 3.2 \\
D: 4.9\end{array}$ & $\begin{array}{l}C:-0.06 \\
D:-0.09\end{array}$ & 2. NA & 2. NA & CD: $13.3(4.3)$ & CD: 35.4 & CD: 39.3 \\
\hline D776K:KA & NA & NA & NA & NA & $\begin{array}{l}A B: 12.4(3.5) \\
C D: 12.4(3.5)\end{array}$ & $\begin{array}{l}A B: 35.0 \\
C D: 32.8\end{array}$ & $\begin{array}{l}A B: 38.9 \\
C D: 36.9\end{array}$ \\
\hline
\end{tabular}

NA, Not applicable; ND, no rigid-body motion reported by dyndom.

*Values are for comparisons made within the same crystal form only (i.e., lo vs 10 and Po vs Po).

${ }^{\dagger}$ Lys544 precedes the GlyThr linker in the LBD construct, and M1 in full-length GluK2. Pro667 follows the linker in the LBD construct, and M3 in full-length GluK2.

${ }^{\ddagger} \mathrm{NCS}$ restraints were used in refinement of GluK2-D776K:KA. Cleft-closure comparisons were therefore made with protomer A only.

the more open cleft in the KA complexes; if the dimer interface had not changed in the KA complexes the movement in the linkers relative to the Glu complexes would have been 2-3 times larger.

Comparing GluK2-D776K dimers with Glu or KA bound also revealed domain movements of a similar magnitude (Fig. 5C; Table 2). Unlike in GluK2-WT and GluK2-M770K, where the direction of the rotational axis resulted in a twist of the dimer interface, in GluK2-D776K the binding of KA induced a hinged motion, increasing the separation between linker residues and bringing the D1 apices closer together compared with the Glu complex (Table 2). Again, in terms of gating this motion would be expected to increase the relative efficacy of KA. Overall, these comparisons therefore identified coherent mutation- and ligandspecific shifts in the dimer interface in GluK2-WT, GluK2M770K, and GluK2-D776K that significantly affected the positions of the linker residues leading to the pore.

\section{Discussion}

\section{Dimer stabilization in the GluK2-D776K mutant}

This study was initially undertaken to test the hypothesis that the lysines introduced in the M770K and D776K mutants act as surrogate cations (Wong et al., 2007; Nayeem et al., 2009), replacing the sodium ions normally bound within the GluK2 LBD dimer interface (Plested et al., 2008). Our structural models confirmed this (Fig. 3), but raised a number of additional questions. For the D776K mutation, the main one is what causes the observed stabilization of the LBD dimer (Nayeem et al., 2009), and by inference the block of macroscopic desensitization. The GluK2D776K LBD structures reveal four potential factors; the new inter-protomer contacts formed by Lys776, the anti-parallel stacked conformation adopted by Arg775, the narrowing of the gap between protomers at the dimer apex and the off-axis binding of chloride.

The altered chloride binding appears not to play a major role in dimer stability, as its replacement by either fluoride or nitrate does not disrupt the LBD dimer. Of the others, the new interprotomer contacts formed by Lys776 will certainly increase dimer stability, and are probably the main stabilizing factor. The Arg775-Arg775 interaction may also contribute: Direct contacts between arginines are not uncommon in protein interfaces (Magalhaes et al., 1994; Pednekar et al., 2009), including in other iGluR LBD dimers (Greger et al., 2006; Naur et al., 2007), and are thought to be stabilized by cation- $\pi$ interactions. For the third potential stabilizing factor, closer apical approach, we cannot distinguish cause and effect. The Arg775 interaction may either contribute to the narrowing of the gap between protomers, or be a consequence of it. Similarly, while movement of the apex is not necessary for Lys776 to bridge the gap, the side-chain methylene groups may help stabilize a closer apical approach through Van der Waal forces or entropic effects. Whatever the relative balance of factors, it is likely that closer packing at the dimer apex in GluK2-D776K does contribute to overall dimer stability.

\section{Role of ions in dimer stability and macroscopic desensitization}

In GluK2-D776K, chloride binding does not appear to contribute significantly to dimer stabilization. This contrasts with data showing that cation and anion binding stabilizes the LBD dimer of a GluK2 mutant with attenuated desensitization (Chaudhry et al., 2009a). It therefore leaves open the question of why GluK2D776K responses are significantly reduced in fluoride and nitrate (Nayeem et al., 2009), implying a functional requirement for chloride binding beyond dimer stabilization. The anion-binding pocket in GluK2-D776K is less well defined than that in GluK2WT, with at least one and possibly more partially occupied sites. This is despite the more electropositive charge-balance, which might have been expected to promote chloride binding. Changes to the shape of the binding pocket, and particularly to Arg775, probably account for the weaker chloride binding. It seems likely that binding of other anions would be similarly affected, resulting in the observed anion-selectivity at a functional level. How this differential anion binding might affect function will require further investigation.

Similar questions can be asked about the role of ions in the GluK2-M770K mutant phenotype. Displacement of sodium ions neatly explains the cation insensitivity, but not the anioninsensitivity or faster macroscopic desensitization. Cations and anions have been suggested to interact with kainate receptors either through a dipole (Wong et al., 2007) or an allosteric cou- 
pling (Plested et al., 2008), but in practice the distinction is a fine one. In both models cation binding alters the electrostatic field at the dimer interface, promoting anion binding. As the sodium ion makes almost no inter-protomer contacts (Plested et al., 2008), dimer stabilization therefore should depend principally on binding of chloride. By extension, faster current decay in GluK2-M770K would be expected to be associated with weak or absent chloride binding, a hypothesis supported by the observation that, unlike GluK2-WT, this mutant is functional in the complete absence of monovalent ions (Wong et al., 2006). Our finding that chloride binding is unaffected by the $\mathrm{M} 770 \mathrm{~K}$ mutation appears at odds with this.

If reduced chloride binding does not explain the GluK2-M770K phenotype, then faster current decay must result from the displacement of sodium by lysine. Cations must therefore do more than simply stabilize the LBD dimer by promoting the binding of anions. There is some evidence for this. First, it has been proposed that the lack of KA receptor responses in the absence of external ions is the result of entry into an inactive state, distinct from desensitization (Wong et al., 2006; Bowie, 2010). Second, the occupancies of a range of monovalent cations show some discrimination in GluK2-WT LBD structures $\left(\mathrm{Li}^{+} / \mathrm{Na}^{+} / \mathrm{K}^{+}=1.0 ; \mathrm{Rb}^{+} / \mathrm{Cs}^{+}=\right.$ $0.5-0.7)$, but in each case the chloride site is fully occupied (Plested et al., 2008). In GluK2-M770K the cation-binding site appears fully occupied, either by the lysine amine or by $\mathrm{Na}^{+}$. The lack of cross-dimer contacts at this site means that at least part of the greater "efficacy" of sodium ions in stabilizing the dimer (or otherwise promoting an "active" dimer conformation) must result from an as yet unidentified mechanism. Wong et al. hypothesized that the $\mathrm{M} 770 \mathrm{~K}$ lysine mimics a rubidium ion, as the phenotypes are very similar (Wong et al., 2007). The physicochemical properties of rubidium ions and lysines are quite distinct, but both groups effectively introduce a partial positive-charge at this site. Glu524 interacts with sodium, while the neighboring Arg523 interacts with the $\alpha$-carboxyl group of glutamate and other ligands (Fig. 2), making an allosteric or electrostatic link one possibility.

\section{Ligand-specific shifts in dimer conformation}

The model of non-NMDA receptor activation linking LBD conformation to gating was initially established for AMPA receptor subunits (Armstrong and Gouaux, 2000). The extent of cleftclosure is generally a good proxy for efficacy in these subunits, but exceptions have been identified. For example, mutants have been GluK2-WT-D776K:Glu (A).
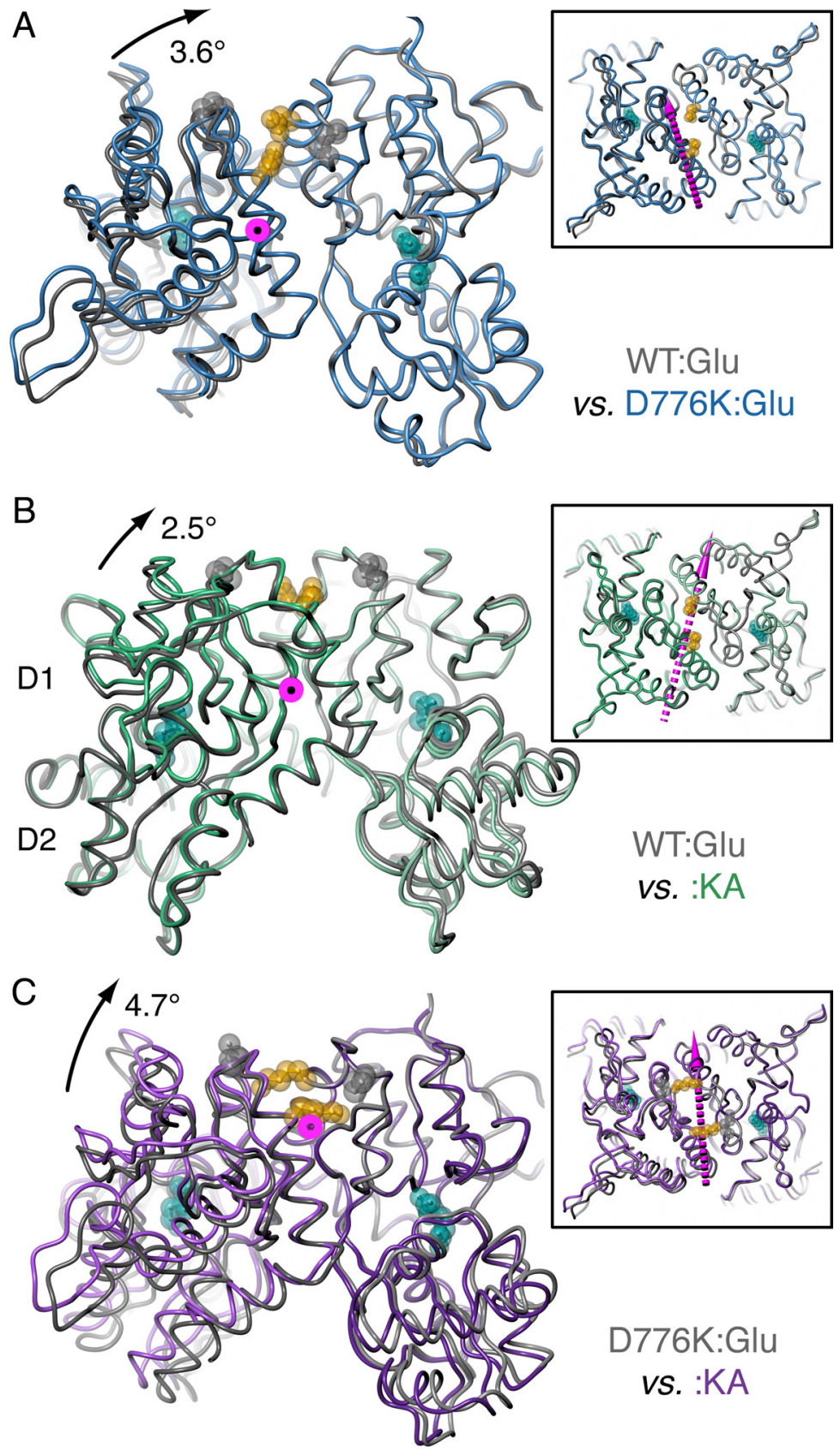

Figure 5. Hinged and pivoted movements in the LBD dimer interface. A, GluK2-WT:Glu (gray) and GluK2-D776K:Glu (lo crystal form; blue) aligned on the D1 domains of protomers A only (right side), to highlight shifts in the dimer interface. The main panel is viewed along the rotational axis (magenta with black center), and the boxed inset is viewed from above the twofold to show vector direction (dashed magenta arrow). For orientation ligand (cyan) and residues 770 (gray, main panel only) and 776 (orange) are shown as sticks/space-fill. B, C, Equivalent views of GluK2-WT:Glu (gray) and GluK2-WT:KA (green) and GluK2-D776K:Glu (I0 crystal form; gray) and GluK2-WT:KA (purple), respectively, aligned and displayed as in $\boldsymbol{A}$. In all parts the magnitude of the rotational component is indicated at the top left, where its effect is most evident. Movement due to cleft-closure is apparent comparing Glu and KA complexes in $\boldsymbol{B}$ and $\boldsymbol{C}$ (main panels, right-side protomer D2 domains), but not comparing GluK2-WT:Glu and

characterized where partial agonists induce complete cleftclosure (Zhang et al., 2008), or where efficacy is related to D2 domain twisting (Birdsey-Benson et al., 2010). For kainate receptor subunits the correlation between cleft-closure and efficacy has also been questioned, with partial agonists again inducing complete cleft-closure in either x-ray structures (Frydenvang et al., 2009) or modeling studies (Fay et al., 2009). In this context, our identification of ligand-specific changes in LBD dimer conformation in the GluK2 
kainate receptor subunit is interesting, as they have the potential to affect receptor gating independently of cleft closure.

The dimer interface has tended to be viewed as a switch with two states; associated/active and dissociated/desensitized. However, gating in non-NMDA receptors involves multiple resting, active and desensitized/inactive states (Robert and Howe, 2003). Our observation of conformational flexibility in the dimer interface opens the possibility that more subtle changes in dimer orientation underlie some of these functional states. The rotational shifts occurring between Glu- and KA-bound LBD dimers were small, but of a magnitude similar to the difference in cleft closure. Their effect may therefore be significant; in both GluK2-WT and GluK2-M770K the direction and magnitude of this "dimer pivot" served to counteract the cleft-closure motion (Fig. 5). As a consequence, two common proxies for agonist efficacy, cleft closure and linker separation, were partially decoupled. In GluK2-WT (and GluK2-M770K), the dimer interface pivots on an axis that intersects the twofold just below the chloride ion. The structural driver for the movement is still to be identified. One possibility is direct coupling between the ligand-binding pocket and the cation-binding site (and dimer interface), as discussed above. Alternatively, there might be a less direct link through Tyr488, which is the only binding site residue in the D1 domain to move upon KA binding (Fig. 4).

The dimer conformation also changes in GluK2-D776K, but in this case the mutation itself is the dominant factor. As discussed above, apical residues are brought closer together by the stronger contacts formed in this region. Interestingly a hinged motion along a similar axis, but moving the apical residues further apart, has been proposed to underlie promotion of a nonconducting state in the GluA2-S729C mutant (Armstrong et al., 2006). Block of macroscopic desensitization cannot be assumed to result from blocked entry into desensitized states (Bowie, 2010). It is, however, interesting that GluA2-S729C and GluK2D776K, mutants with contrasting phenotypes, are associated with opposing movements at the LBD dimer interface.

In the KA complex the hinged movement is even more pronounced. This conformational difference between Glu and KA complexes can be correlated with two properties of the GluK2D776K mutant (Nayeem et al., 2009). First, the relative efficacy of $\mathrm{KA}$ is higher in the mutant (0.86) than in GluK2-WT (0.62), consistent with the observed linker spacing (Table 2). Notably, the extent of cleft closure induced by KA is unaffected by the D776K mutation. Second, the tighter dimer association in the KA complex may underlie the slower deactivation kinetics of GluK2D776K responses to $\mathrm{KA}$, with the dimer conformation representing a "supra-active" state. This would in turn slow channel closure on removal of ligand, a further indication that relatively small changes in dimer conformation may prove to define the differences between iGluR functional states.

\section{References}

Adams PD, Grosse-Kunstleve RW, Hung LW, Ioerger TR, McCoy AJ, Moriarty NW, Read RJ, Sacchettini JC, Sauter NK, Terwilliger TC (2002) PHENIX: building new software for automated crystallographic structure determination. Acta Crystallogr D Biol Crystallogr 58:1948-1954.

Armstrong N, Gouaux E (2000) Mechanisms for activation and antagonism of an AMPA-sensitive glutamate receptor: crystal structures of the GluR2 ligand binding core. Neuron 28:165-181.

Armstrong N, Jasti J, Beich-Frandsen M, Gouaux E (2006) Measurement of conformational changes accompanying desensitization in an ionotropic glutamate receptor. Cell 127:85-97.

Baker NA, Sept D, Joseph S, Holst MJ, McCammon JA (2001) Electrostatics of nanosystems: application to microtubules and the ribosome. Proc Natl Acad Sci U S A 98:10037-10041.
Birdsey-Benson A, Gill A, Henderson LP, Madden DR (2010) Enhanced efficacy without further cleft closure: reevaluating twist as a source of agonist efficacy in AMPA receptors. J Neurosci 30:1463-1470.

Bowie D (2010) Ion-dependent gating of kainate receptors. J Physiol 588:67-81.

CCP4 [Collaborative Computational Project, Number 4] (1994) The CCP4 suite: programs for protein crystallography. Acta Crystallogr D Biol Crystallogr 50:760-763.

Chaudhry C, Plested AJ, Schuck P, Mayer ML (2009a) Energetics of glutamate receptor ligand binding domain dimer assembly are modulated by allosteric ions. Proc Natl Acad Sci U S A 106:12329-12334.

Chaudhry C, Weston MC, Schuck P, Rosenmund C, Mayer ML (2009b) Stability of ligand-binding domain dimer assembly controls kainate receptor desensitization. EMBO J 28:1518-1530.

Das U, Kumar J, Mayer ML, Plested AJ (2010) Domain organization and function in GluK2 subtype kainate receptors. Proc Natl Acad Sci U S A 107:8463-8468.

Emsley P, Lohkamp B, Scott WG, Cowtan K (2010) Features and development of Coot. Acta Crystallogr D Biol Crystallogr 66:486-501.

Fay AM, Corbeil CR, Brown P, Moitessier N, Bowie D (2009) Functional characterization and in silico docking of full and partial GluK2 kainate receptor agonists. Mol Pharmacol 75:1096-1107.

Frydenvang K, Lash LL, Naur P, Postila PA, Pickering DS, Smith CM, Gajhede M, Sasaki M, Sakai R, Pentikaïnen OT, Swanson GT, Kastrup JS (2009) Full domain closure of the ligand-binding core of the ionotropic glutamate receptor iGluR5 induced by the high affinity agonist dysiherbaine and the functional antagonist 8,9-dideoxyneodysiherbaine. J Biol Chem 284:14219-14229.

Greger IH, Akamine P, Khatri L, Ziff EB (2006) Developmentally regulated, combinatorial RNA processing modulates AMPA receptor biogenesis. Neuron 51:85-97.

Hayward S, Berendsen HJ (1998) Systematic analysis of domain motions in proteins from conformational change: new results on citrate synthase and T4 lysozyme. Proteins 30:144-154.

Kabsch W (1993) Automatic processing of rotation diffraction data from crystals of initially unknown symmetry and cell constraints. J Appl Crystallogr 26:795-800.

Krissinel E, Henrick K (2007) Inference of macromolecular assemblies from crystalline state. J Mol Biol 372:774-797.

Magalhaes A, Maigret B, Hoflack J, Gomes JN, Scheraga HA (1994) Contribution of unusual arginine-arginine short-range interactions to stabilization and recognition in proteins. J Protein Chem 13:195-215.

Mayer ML (2005) Crystal structures of the GluR5 and GluR6 ligand binding cores: molecular mechanisms underlying kainate receptor selectivity. Neuron 45:539-552.

Mayer ML (2006) Glutamate receptors at atomic resolution. Nature 440:456-462.

McCoy AJ, Grosse-Kunstleve RW, Adams PD, Winn MD, Storoni LC, Read RJ (2007) Phaser crystallographic software. J Appl Crystallogr 40:658-674.

Naur P, Hansen KB, Kristensen AS, Dravid SM, Pickering DS, Olsen L, Vestergaard B, Egebjerg J, Gajhede M, Traynelis SF, Kastrup JS (2007) Ionotropic glutamate-like receptor delta2 binds D-serine and glycine. Proc Natl Acad Sci U S A 104:14116-14121.

Nayeem N, Zhang Y, Schweppe DK, Madden DR, Green T (2009) A nondesensitizing kainate receptor point mutant. Mol Pharmacol 76:534-542.

Paternain AV, Cohen A, Stern-Bach Y, Lerma J (2003) A role for extracellular $\mathrm{Na}+$ in the channel gating of native and recombinant kainate receptors. J Neurosci 23:8641-8648.

Pednekar D, Tendulkar A, Durani S (2009) Electrostatics-defying interaction between arginine termini as a thermodynamic driving force in protein-protein interaction. Proteins 74:155-163.

Plested AJ, Mayer ML (2007) Structure and mechanism of kainate receptor modulation by anions. Neuron 53:829-841.

Plested AJ, Vijayan R, Biggin PC, Mayer ML (2008) Molecular basis of kainate receptor modulation by sodium. Neuron 58:720-735.

Pražnikar J, Afonine PV, Gunčar G, Adams PD, Turk D (2009) Averaged kick maps: less noise, more signal. and probably less bias. Acta Crystallogr D Biol Crystallogr 65:921-931.

Priel A, Selak S, Lerma J, Stern-Bach Y (2006) Block of kainate receptor desensitization uncovers a key trafficking checkpoint. Neuron 52: 1037-1046. 
Robert A, Howe JR (2003) How AMPA receptor desensitization depends on receptor occupancy. J Neurosci 23:847-858.

Sobolevsky AI, Rosconi MP, Gouaux E (2009) X-ray structure, symmetry and mechanism of an AMPA-subtype glutamate receptor. Nature 462:745-756.

Stern-Bach Y, Russo S, Neuman M, Rosenmund C (1998) A point mutation in the glutamate binding site blocks desensitization of AMPA receptors. Neuron 21:907-918.

Studier FW (2005) Protein production by auto-induction in high density shaking cultures. Protein Expr Purif 41:207-234.

Sun Y, Olson R, Horning M, Armstrong N, Mayer M, Gouaux E (2002) Mechanism of glutamate receptor desensitization. Nature 417:245-253.

Weston MC, Schuck P, Ghosal A, Rosenmund C, Mayer ML (2006) Con- formational restriction blocks glutamate receptor desensitization. Nat Struct Mol Biol 13:1120-1127.

Wong AY, Fay AM, Bowie D (2006) External ions are coactivators of kainate receptors. J Neurosci 26:5750-5755.

Wong AY, MacLean DM, Bowie D (2007) $\mathrm{Na}^{+} / \mathrm{Cl}^{-}$dipole couples agonist binding to kainate receptor activation. J Neurosci 27:6800-6809.

Zhang W, Cho Y, Lolis E, Howe JR (2008) Structural and single-channel results indicate that the rates of ligand binding domain closing and opening directly impact AMPA receptor gating. J Neurosci 28: 932-943.

Zhang Y, Nayeem N, Nanao MH, Green T (2006) Interface interactions modulating desensitization of the kainate-selective ionotropic glutamate receptor subunit GluR6. J Neurosci 26:10033-10042. 